\title{
Prediction of Transonic Duct Flow Using a Zonal Hybrid RANS-LES Modeling Approach
}

\author{
Sebastian Arvidson, Shia-Hui Peng and Lars Davidson
}

\begin{abstract}
Transonic duct flow with shock/boundary-layer interaction (SBLI) was analyzed using a zonal hybrid RANS-LES approach. The proposed zonal approach simulates the attached boundary layer flow, prior to the SBLI region in RANS mode. At a prescribed streamwise location, upstream of the SBLI region, the model switches to its hybrid RANS-LES mode over a buffer zone in order to avoid possible discontinuities. The corner separation bubbles, induced by the shock at $\mathrm{M}=1.4$, were mostly simulated in LES mode in order to improve the SBLI flow prediction. In addition to comparisons with experimental data, the zonal approach is compared to simulations using the SA-DDES and SA-IDDES model.

The zonal approach predicted a corner separation bubble and a $\lambda$-shape shock which is in good agreement with experimental data. Furthermore, the predicted pressure rise across the shock agrees reasonably well with the experiment.
\end{abstract}

\section{Introduction}

Hybrid RANS-LES modeling approaches have been increasingly used in aeronautic applications for better predictions of unsteady and complex aerodynamic flows. Facilitated by this, hybrid RANS-LES methods have been extensively studied and verified in simulations of a large variety of flows. At an early stage, the development of hybrid RANS-LES methods focused most on high-Re number turbulent flows with massive separation, such as the flow over an aircraft wing at high incidence or

Sebastian Arvidson, Shia-Hui Peng, Lars Davidson

Division of Fluid Dynamics, Chalmers University of Technology, SE-412 96 Gothenburg, Sweden, e-mail: sebastian.arvidson, peng, lada@chalmers.se

Sebastian Arvidson

Saab Aeronautics, SE-581 88 Linköping, Sweden, e-mail: sebastian.arvidson@ saabgroup.com

Shia-Hui Peng

Swedish Defence Research Agency (FOI), SE-164 90 Stockholm, Sweden, e-mail: peng@foi.se 
over other bluff bodies. With increasing computer capacities and refined modeling approaches, hybrid RANS-LES methods have shown promising advantages in the computational analysis of unsteady boundary layer separation. This has motivated the present study, verifying hybrid RANS-LES methods for a SBLI flow.

SBLI flows have been commonly encountered in aeronautical applications and appears in such phenomena as buffeting on aircraft wings and in high-speed inlet configurations. For high-speed inlets, SBLI can typically take place inside the inlet duct, which can have a severe impact on the engine/inlet stability if it is not controlled. SBLI flow in a duct often causes flow separation in the corners of the duct. However, predicting SBLI in duct flows using CFD methods has been found to be challenging, especially using hybrid RANS-LES and DES methods [1, 3].

This paper analyzes a transonic duct flow involving SBLI [4]. A rectangular duct with a convergent-divergent nozzle was used to accelerate the flow from subsonic speed to supersonic speed, where a shock takes place at Mach 1.4. The $\lambda$-shape shock wave interacts with the boundary layer and consequently leading to local recirculation bubbles in the duct corners as shown in Fig. 1. The walls are parallel in the shock region and the cross-sectional area is constant with $H=178 \mathrm{~mm}$ and $W=117 \mathrm{~mm}$ in height and width, respectively. The center line length of the duct is $L=1030 \mathrm{~mm}$. The computational domain can be seen in Fig. 2 .

Bottom wall pressures and streamwise velocities in the duct center plane are available from the experiment [4]. A comparison is made of profiles from the simulations and the experiment at a distance $\Delta x$ relative to the shock location, as illustrated in Fig. 1. Schlieren photographs of the shock wave and oil-flow visualization of the bottom wall flow are also available and used for comparison. These are shown in Fig. 1. Measured at the center line of the duct, the shock wave in the experiment occurs at $x=659 \mathrm{~mm}(\Delta x=0)$, i.e. $205 \mathrm{~mm}$ downstream of the nozzle exit. The Reynolds number, based on the displacement thickness and the local freestream velocity, $U_{0}$, at $\Delta x=-30 \mathrm{~mm}$ is $\operatorname{Re}_{\delta^{*}}=13600$.

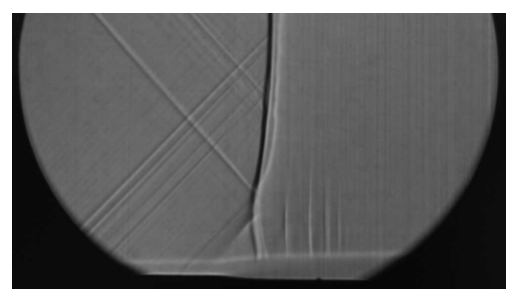

(a) Shock wave with $\lambda$-foot.

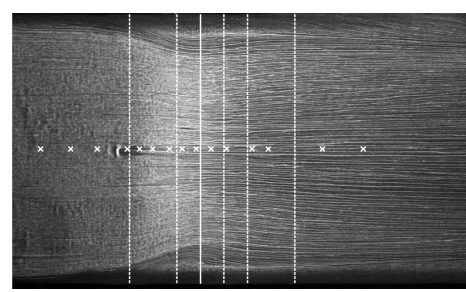

(b) Oil-flow visualization.

Fig. 1 Experimental visualization of (a) shock wave and (b) shock induced boundary layer separation. In (b), lines indicate the locations of the velocity profiles from the shock at $\Delta x=-30,-10$, $0,10,20$ and $40 \mathrm{~mm}$. Crosses indicate locations where wall pressures were measured.

For the transonic duct flow involving SBLI analyzed in this paper, conventional hybrid RANS-LES, DES and RANS approaches fail to predict the SBLI flow field $[3,5]$. It is shown that the response of modeling to the onset of the corner sep- 
aration bubble is one of the key issues for an accurate prediction of the shock wave and the subsequent SBLI flow. It is further revealed that an accurate prediction of the shock intensity is closely associated with the modeling capability and gives significant improvements on the prediction of the overall SBLI flow properties.

This work is a continuation of a previous work presented in [3] to improve the modeling and further to explore the underlying physics of SBLI with an improved prediction. The paper presents a zonal hybrid RANS-LES modeling approach based on a low-Re number $k-\omega$ model, which was applied to the transonic duct flow. Moreover, an extensive analysis is given of the simulations using the zonal approach. In addition to the zonal approach, SA-model [10] based DDES [12] and IDDES [11] were also used to simulate the flow. RANS results with the low-Re number $k-\omega$ model are also included for reference.

\section{Zonal hybrid RANS-LES modeling}

The PDH-LRN $k-\omega$ model by Peng et. al [9] (hereafter PDH-LRN) was shown in [3] to give reasonably accurate RANS results for the flow analyzed. Moreover, it was shown in [3] that RANS simulations using the Spalart-Allmaras model [10] and the Menter SST $k-\omega$ model [7] fail to give symmetric corner separation bubbles leading to a collapsed shock. The PDH-LRN is therefore used as the base model in the zonal hybrid RANS-LES modeling approach (hereafter ZHYBRID) used in this paper. The $k$ and $\omega$ transport equations for the PDH-LRN model read:

$$
\begin{gathered}
\frac{D \rho k}{D t}=\tau_{i j} \frac{\partial u_{i}}{\partial x_{j}}-D^{k}+\frac{\partial}{\partial x_{j}}\left[\left(\mu+\frac{\mu_{t}}{\sigma_{k}}\right) \frac{\partial k}{\partial x_{j}}\right] \\
\frac{D \rho \omega}{D t}=C_{\omega_{1}} f_{\omega} \frac{\omega}{k} \tau_{i j} \frac{\partial u_{i}}{\partial x_{j}}-C_{\omega 2} \rho \omega^{2} \\
+\frac{\partial}{\partial x_{j}}\left[\left(\mu+\frac{\mu_{t}}{\sigma_{\omega}}\right) \frac{\partial \omega}{\partial x_{j}}\right]+C_{\omega} \frac{\mu_{t}}{k} \frac{\partial k}{\partial x_{j}} \frac{\partial \omega}{\partial x_{j}} \\
\mu_{t}=C_{\mu} f_{\mu} \frac{\rho k}{\omega}
\end{gathered}
$$

The turbulence-resolving LES mode, based on the PDH-LRN model, is incorporated through the dissipation term in the $k$-equation, see further $[1,2]$.

$$
D^{k}=C_{k} f_{k} \rho k \omega=\rho f_{k} \frac{k^{3 / 2}}{l_{\text {turb }}}
$$

The RANS and LES zones are prescribed for this transonic duct flow as shown in Fig. 2. Three zones have been accordingly defined. The upstream attached boundary layer flow is treated in RANS mode, via a buffer zone, hybrid RANS-LES modeling is then used to accommodate the flow in the SBLI region and further downstream 
to the outflow section. In previous works $[1,2]$ on channel flow, the switch from RANS to LES was made at a prescribed interface plane orthogonal to the streamwise direction. Moreover, the RANS turbulent kinetic energy was manipulated across the interface to match the LES subgrid scale (SGS) turbulent kinetic energy level. Thus, the turbulent viscosity at the interface was reduced from a RANS level to a SGS level. However, in this work we instead use a buffer zone for the turbulent length scale according to Eqs. (5)-(7), which has additionally been defined in between the RANS and the hybrid RANS-LES zones. The buffer zone helps to eliminate possible discontinuities in the transition from RANS to LES mode and is patched between $x_{b 1}$ and $x_{b 2}$ in the streamwise direction upstream of the shock. In this paper $x_{b 1}=470$ $\mathrm{mm}$ and $x_{b 2}=520 \mathrm{~mm}$, see Fig. 2 and 4 .

$$
\begin{gathered}
l_{b}=f_{b} l_{L E S}+\left(1-f_{b}\right) l_{R A N S} \\
f_{b}=\tanh \left(\left[C_{b 1} r_{b}\right]^{C_{b 2}}\right), C_{b 1}=2, C_{b 2}=3 \\
r_{b}=\frac{x-x_{b 1}}{x_{b 1}-x_{b 2}}, x_{b 1} \leq x \leq x_{b 2}
\end{gathered}
$$

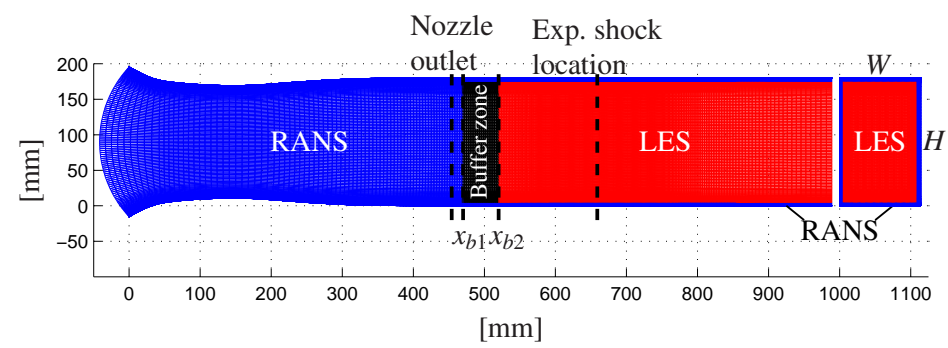

Fig. 2 Computational grid with RANS and LES zones used in the ZHYBRID simulations. Blue: RANS; black: buffer zone; red: LES. Left grid: xy-plane, flow from left to right. Right grid: yzplane, grid cross-section.

The RANS-LES interface in the near-wall layer for hybrid RANS-LES modeling is predefined at a distance of $d_{w}=d_{w s}$ from the wall, where $d_{w}$ is the wall distance and $d_{w s}$ is the distance from the wall at which the RANS-LES switch is located. In this paper $d_{w s}=5 \mathrm{~mm}\left(d_{w s} / H=0.028\right)$. The turbulent length scale used in the zonal approach is defined as:

$$
l_{t u r b}= \begin{cases}l_{R A N S} & \text { if } x<x_{b 1} \\ l_{R A N S} & \text { if } d_{w} \leq d_{w s} \\ l_{b} & \text { if } x_{b 1} \leq x \leq x_{b 2} \text { and } d_{w}>d_{w s} \\ l_{L E S} & \text { if } x>x_{b 2} \text { and } d_{w}>d_{w s}\end{cases}
$$




$$
l_{R A N S}=\frac{k^{1 / 2}}{c_{k} \omega}, l_{L E S}=\Psi_{P D H} C_{L E S} \Delta_{d w}
$$

$$
\Delta_{d w}=\min \left(\max \left[c_{w} d_{w}, c_{w} \Delta_{\max }\right], \Delta_{\max }\right), \Delta_{\max }=\max \left(\Delta_{x}, \Delta_{y}, \Delta_{z}\right), c_{w}=0.15
$$

The LES length scale, $\Delta_{d w}$, was originally used in the IDDES formulation [11]. However, compared to the original formulation, the grid step in the wall normal direction is omitted in the ZHYBRID model in the max-argument in Eq. (10). This modification was also used in [8]. The details in the hybrid RANS-LES model can be found in [2].

\section{Computational setup}

The simulations presented were performed with the unstructured compressible Navier-Stokes solver Edge [6] on a hexahedral grid using $\left(n_{x \max }, n_{y \max }, n_{z \max }\right)=$ $(306,180,136)$ grid points. The time-marching was done using an implicit secondorder backward Euler scheme with a time step of $\Delta t=4 \times 10^{-6} \mathrm{~s}$. This gave a maximum convective CFL number of approximately 1 . At each time step, 100 to 200 sub-iterations have been conducted to converge the solution using a three-stage Runge-Kutta scheme and an algebraic multigrid method. The momentum and turbulent transport equations were discretized using a second-order central differencing scheme.

On the inlet boundary, total pressure $\left(p_{0, \text { in }}=147.5 \mathrm{kPa}\right)$, total temperature $\left(T_{0, \text { in }}=293 \mathrm{~K}\right)$ and the flow direction were specified according to the experiment. A turbulence intensity of one percent and $\mu_{t} / \mu=1$ were specified to set the inlet boundary conditions for the turbulent quantities. On the outlet boundary a static pressure, $p_{\text {out }}$, was specified. It is noted here that the predicted location of the shock wave is very sensitive to the specified outlet pressure as seen in Table 1. As expected, a relatively large outlet pressure often leads to an early formation of the shock wave. The outlet pressure is not available from the experiment.

Table 1 Summary of simulations. $\Delta x_{\text {shock }}$ and $\Delta x_{\text {nozzle }}$ indicate time-averaged values.

\begin{tabular}{llll}
\hline Case & $P_{\text {out }}[\mathrm{Pa}]$ & $\Delta x_{\text {shock }}^{*}[\mathrm{~mm}]$ & $\Delta x_{\text {nozzle }}^{* *}[\mathrm{~mm}]$ \\
\hline ZHYBRID-a & 84000 & -84 & 121 \\
ZHYBRID-b & 78500 & 0 & 205 \\
SA-DDES & 83000 & +61 & 266 \\
SA-IDDES & 83250 & +26 & 231 \\
PDH-LRN (RANS) & 84000 & +2 & 207 \\
\hline
\end{tabular}

${ }^{*}$ Simulated shock location in relation to experimental location: $\Delta x_{\text {shock }}=x_{\text {shock,sim }}-x_{\text {shock,exp }}$.

${ }^{* *}$ Simulated shock location in relation to nozzle outlet location: $\Delta x_{\text {nozzle }}=x_{\text {shock,sim }}-x_{\text {nozzle }}$. 
In the simulations 8000-16000 time steps were needed to establish a fullydeveloped turbulence-resolving SBLI flow field from the initial RANS flow field. Another 8000 time steps, which represents a fluid particle passing through the duct approximately ten times, were used for the statistical analysis.

In relation to the modeling approach and the outlet pressure, $p_{\text {out }}$, specified, the offset, $\Delta x_{\text {shock}}$, between the predicted and the measured shock location is presented in Table 1 .

Two simulations using the ZHYBRID model are presented, one with the same outlet pressure used as in the PDH-LRN RANS simulation and another with the outlet pressure being adjusted to agree with the experimental shock location. The PDH-LRN RANS simulation is also included in Table 1 for reference. For the SADDES and SA-IDDES computations $p_{\text {out }}=83000 \mathrm{~Pa}$ and $83250 \mathrm{~Pa}$ were used, respectively. Applying the SA-RANS model to this transonic duct flow gives an asymmetric flow field and a weak shock [3]. However, the shock location at the duct center line agrees reasonably well with experimental data for $p_{\text {out }}=83000 \mathrm{~Pa}$, which has motivated the choice of outlet pressure in the SA-DDES and SA-IDDES computations.

\section{Results and discussion}

As seen in Table 1 and in Fig. 3 (a), the PDH-LRN RANS simulation using $p_{\text {out }}=$ 84000 Pa reproduces well the shock location and the shock intensity, i.e. the pressure rise across the shock. Applying the outlet pressure $p_{\text {out }}=84000 \mathrm{~Pa}$ to the ZHYBRID model (denoted ZHYBRID-a), the simulated shock location is shifted upstream as compared to the PDH-LRN RANS simulation and to the experiments. Moreover, a weak secondary shock is observed. Nevertheless, the shock intensity in terms of the pressure distribution over the shock is reasonably comparable to the experimental data as shown in Fig. 3.

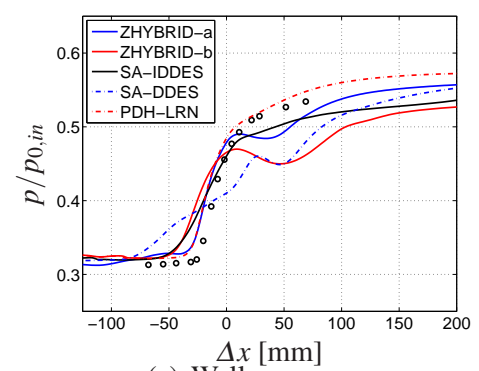

(a) Wall pressure.

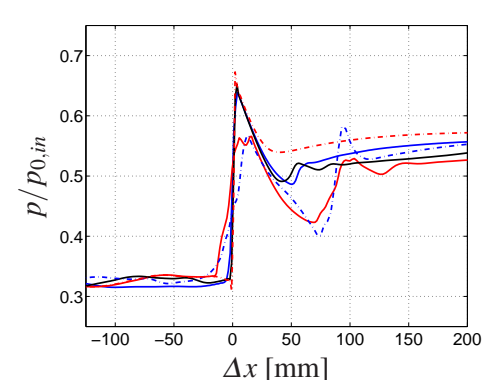

(b) Center line pressure.

Fig. 3 Time-averaged static pressure across the shock. (a) Surface pressure on bottom wall. (b) Pressure along the duct center line. Markers are experimental data. 
As observed in Fig. 4, which shows surface streamlines on the bottom wall in the SBLI region, the predicted corner separation bubbles using the ZHYBRID model are larger than in the PDH-LRN RANS simulation. Moreover, in both ZHYBRID simulations, cross flow is observed downstream of the shock, moving from the side walls into the duct center part, which is not present in the PDH-LRN RANS simulation. This is particularly the case for the ZHYBRID-a simulation. The larger corner separation bubble and the subsequent cross flow observed in the ZHYBRID simulations have reduced the skin friction as compared to the PDH-LRN simulation. Since the same outlet pressure was used in the ZHYBRID-a and PDH-LRN RANS simulations, the shock moved upstream in the ZHYBRID-a case.

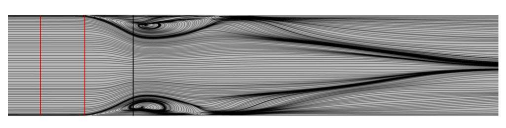

(a) ZHYBRID-a

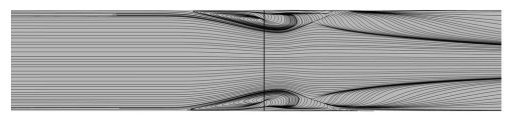

(c) SA-DDES

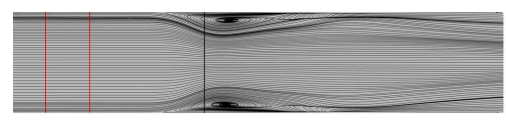

(b) ZHYBRID-b

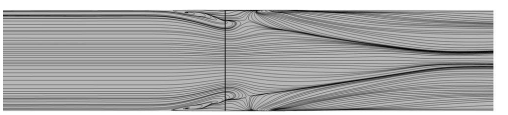

(d) SA-IDDES

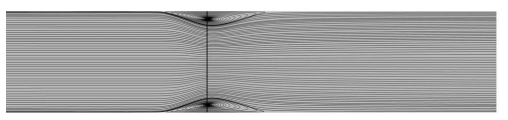

(e) PDH-LRN

Fig. 4 Time-averaged surface streamlines on the bottom wall around the shock. The black line indicates shock location, and the red lines indicate the buffer zone used in ZHYBRID.

The second ZHYBRID simulation presented in Table 1 (denoted ZHYBRID-b) was given a lower outlet pressure in order to match the experimental shock location. As shown in Fig. 3(a), a weaker shock intensity is obtained as expected. Figure 3(b), which shows the time-averaged static pressure across the shock along the duct center line, indicates further that the main pressure peak is much reduced and much less sharp as compared to the ZHYBRID-a result. Moreover, the secondary shock, downstream of the main shock, is more intensive in the ZHYBRID-b simulation.

With a lower outlet pressure and weaker shock intensity, the time-averaged corner separation bubble is longer as compared to that in the ZHYBRID-a computation, as seen in Fig. 4(a) and (b). From the analyses of a series of instantaneous snapshots of the ZHYBRID and SA-IDDES flow fields, it was observed that the corner bubble onset obtained with the ZHYBRID-b and SA-IDDES computations fluctuate over a longer distance in the streamwise direction than the ZHYBRID-a simulation. As an effect of the streamwise fluctuation of the bubble onset, a less steep pressure growth, as shown in Fig. 3(a), and a reduced near wall velocity, which can be seen in in Fig. 6, are observed with the ZHYBRID-b and SA-IDDES cases compared to the ZHYBRID-a and PDH-LRN cases. Moreover, it was noticed that the corner separation bubble shifts from side to side in the ZHYBRID-b simulation. 
A strong fluctuation in the onset of the corner separation bubbles was also observed in the SA-DDES case. The time-averaged pressure distribution in Fig. 3 for the SA-DDES computation indicates an obvious shock collapse near the wall with only a marginal pressure rise. Moreover, the SA-DDES computation has claimed a rather intensive secondary shock over the duct center, as shown in Fig. 3(b).

It is noted that an exaggerated corner separation bubble may lead to a contracted flow through the duct in the form of a fluidic convergent-divergent nozzle. After the velocity retardation across the shock, the flow may become accelerated to supersonic speed downstream of the shock. This may cause secondary shock patterns. This has been the case in the hybrid RANS-LES simulations, but not in the RANS computation, as shown in Fig. 3(b). With ZHYBRID-a and SA-IDDES only a weak secondary shock is observed in the center plane. These secondary shocks are not always present in the resolved instantaneous flow field, but appears when the cross flow towards the duct center is strong and contracts the effective duct flow area downstream of the shock.

The difference seen in the pressures at $\Delta x=200$ in Fig. 3 is partly due to the different back pressures applied at the outlet boundary. Since the cross flow from the side walls towards the duct center is over-predicted, the contraction of the flow contributes to a higher velocity in the duct center region and a lower static pressure than in the experiments. Further downstream, however, it was found that the pressure recovers to the level specified at the boundary.

The switch from RANS to LES can be estimated from the turbulent viscosities in the center plane of the duct shown in Fig. 5. The ZHYBRID RANS-LES switch was prescribed and occurred at the same distance from the wall in both simulations. For the SA-DDES and the SA-IDDES model, the RANS-LES switch is regulated by the local flow properties in the near-wall region. As shown in the profiles of the SADDES turbulent viscosity and the streamwise velocity in the center plane, most of the boundary layer has been treated in RANS mode as expected. In the center plane, the RANS-LES interface with the SA-IDDES model occurs closer to the wall as compared to the SA-DDES and the ZHYBRID model. This may suggest that SAIDDES is in wall-modeled LES mode and has resolved a part of the boundary layer.

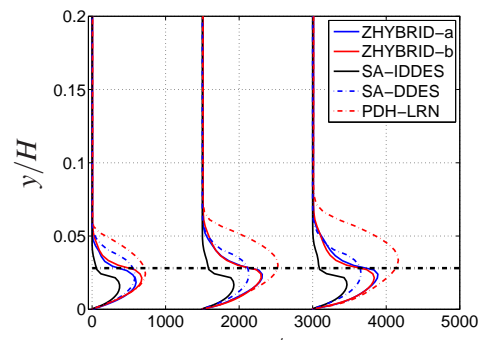

(a) $\Delta x=-30,-\mu_{t} / \mu$

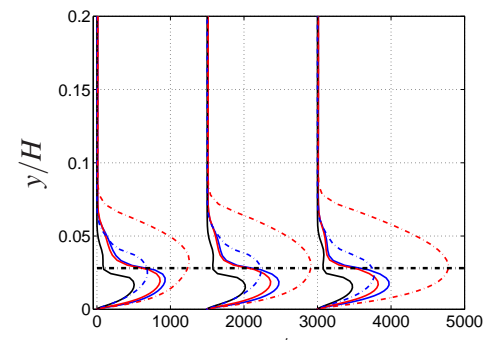

(b) $\Delta x=10,20,40 \mathrm{~mm}$.

Fig. 5 Time-averaged turbulent viscosity in the SBLI region. Dash-dotted horizontal black line indicates ZHYBRID switch from RANS to LES. 


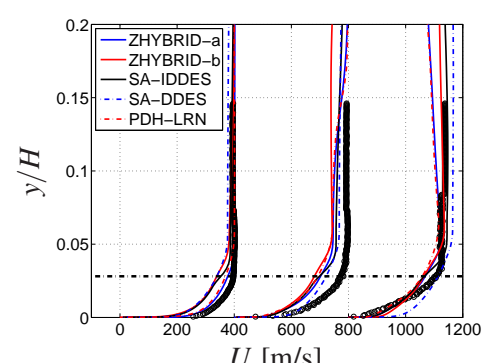

(a) $\Delta x=-30,-10,0 \mathrm{~mm}$.

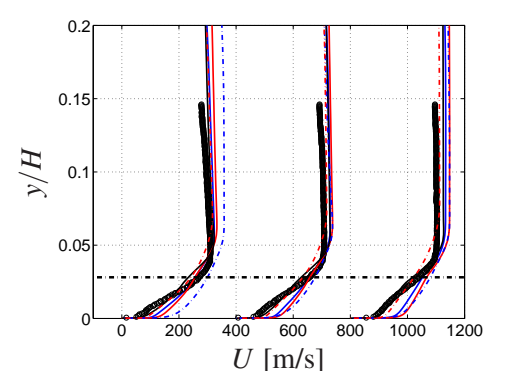

(b) $\Delta x=10,20,40 \mathrm{~mm}$.

Fig. 6 Time-averaged streamwise velocity, $U$, in the SBLI region. Dash-dotted horizontal black line indicates ZHYBRID switch from RANS to LES. Markers are experimental data.

A more rapid reduction of the turbulent viscosity is observed in the SA-IDDES model than in the ZHYBRID model. The RANS-LES switch occurs somewhat farther out from the wall with the ZHYBRID compared to the SA-IDDES and the turbulent viscosity produced by the ZHYBRID model in the near-wall RANS layer is higher, which contributes to the higher level of turbulent viscosity observed in the LES region. Furthermore, the near-wall switch from RANS to LES in the ZHYBRID model does not involve any empirical functions to manipulate the turbulent viscosity across the RANS-LES interface in order to mitigate the log-layer mismatch as in the SA-IDDES model. On the other hand, in $[1,2]$ it was shown in channel flow that the zonal approach based on PDH-LRN has only a weak dependency of the RANS-LES switch location with respect to the log-layer mismatch.

Far upstream at $x=400 \mathrm{~mm}$ (not shown here though), where the effect of the shock is negligible, the velocity profiles of the PDH-LRN and the two ZHYBRID simulations coincide as expected, since the ZHYBRID model is in RANS mode in this flow region. Comparing the SA based models with the PDH-LRN based models at $x=400 \mathrm{~mm}$, the SA based models predicted a slightly fuller profile than the PDHLRN based models.

The shape and width of the corner separation bubbles, as well as the shock foot shape affect significantly the velocity profiles in the center plane of the duct as shown in Fig. 6. The ZHYBRID-a simulation predicts a velocity profile at $\Delta x=-30$ $\mathrm{mm}$ which is in reasonably good agreement with experimental data, indicating a well predicted shock foot and a bubble onset relative to the shock. Moving downstream to $\Delta x=-10 \mathrm{~mm}$, all simulations predict too low a velocity in the near wall region, indicating too early a boundary-layer growth caused by the $\lambda$-shape shock. At the shock, the profiles are close to each other in the near-wall region, except for the SADDES simulation, and compare fairly well with the experimental velocity profile.

The near-wall flow after the shock is best predicted in the SA-IDDES and the PDH-LRN simulations with a slight over-prediction of the velocity in the off-wall region for the SA-IDDES model. At $\Delta x=20$ and $40 \mathrm{~mm}$, both ZHYBRID simulations over-predicts the flow velocity. Moreover, the different shape of the SA-DDES velocity profile at $\Delta x=10 \mathrm{~mm}$, compared to the experiment and to the other simu- 
lations, is an effect of time-averaging the shock motion, which is more pronounced for this simulation.

Large differences in modeling/resolving the flow have been observed when the corner separation flow is analyzed more in details. In Fig. 7, the time-averaged field of turbulent viscosity is overlayed with streamlines of the recirculating corner flow for the models used. In the corner, the SA-DDES model has produced a high level of turbulent viscosity and only very large structures have been resolved as shown in Fig. 8.

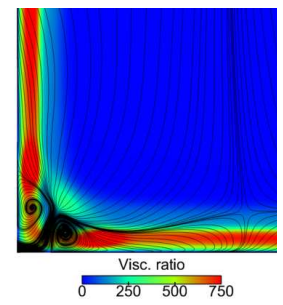

(a) ZHYBRID-a

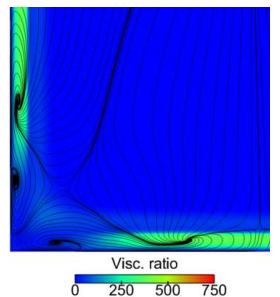

(b) SA-IDDES

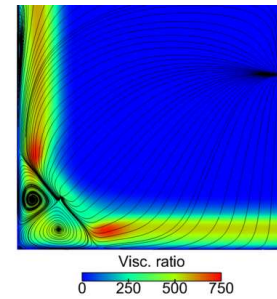

(c) SA-DDES

Fig. 7 Time-averaged streamlines and turbulent viscosity field normalized by molecular viscosity in a cross-sectional cut at $\Delta x=20 \mathrm{~mm}$ in the lower left (aft looking forward) corner separation bubble.

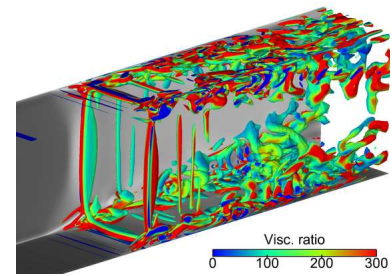

(a) ZHYBRID-a

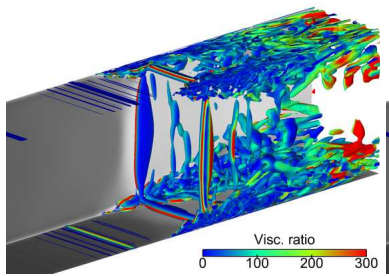

(b) SA-IDDES

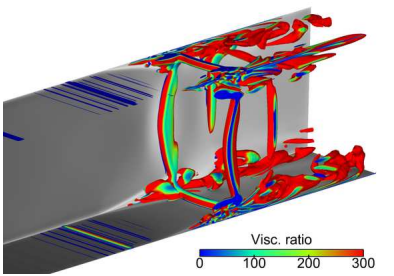

(c) SA-DDES

Fig. 8 Resolved turbulent structures displayed using iso-surfaces of Q-criterion, $Q H^{2} / U_{0}^{2}=1$. Skin friction displayed on the duct walls.

The SA-IDDES model, on the other hand, adapts nicely to the recirculating unsteady flow in the corner separation bubble, switching to LES and resolving reasonably well the core of the recirculations. In the ZHYBRID-a case, the "eye" of the recirculation is located in the prescribed RANS region. However, the outer part of the recirculation region predicted in the ZHYBRID-a simulation is resolved with LES, which gives a higher level of resolved turbulent structures compared to the SA-DDES model as shown in Fig. 8. In an additional simulation using the ZHYBRID model with $d_{w s}=2.5 \mathrm{~mm}$ and $p_{\text {out }}=78500 \mathrm{~Pa}$ (not included in this paper), more resolved turbulence was observed in the corner separation bubble compared to $d_{w s}=5 \mathrm{~mm}$. It is also observed in all simulations that the turbulent structures are 
concentrated in the corner separation bubbles and the cross flow moving from the side walls towards the duct center. Moreover, no resolved turbulence is present in the upstream attached boundary layer in any of the simulations since the attached boundary layer is simulated in RANS mode and the strong fluctuations imposed by the shock have a very weak effect on the flow field upstream of the SBLI region. The concentration of resolved turbulent structures in the SBLI region is thus expected and the aim of the modeling techniques chosen.

Analyzing the ZHYBRID simulations, the blending function used in the buffer zone rapidly forces the ZHYBRID model to switch from RANS to LES without any discontinuities in the turbulent quantities. However, in the ZHYBRID-a simulation, the buffer zone, which is located close to the onset of the corner bubble, delays the formation of resolved turbulence compared to the SA-IDDES computation.

\section{Summary and conclusions}

A zonal hybrid RANS-LES approach (ZHYBRID), based on the PDH-LRN $k-\omega$ model (PDH-LRN), has been applied to a transonic duct flow with shock/boundarylayer interaction. The ZHYBRID simulations have been compared to simulations performed with the Spalart-Allmaras (SA) based DDES and IDDES computations. Moreover, a RANS simulation using the PDH-LRN model has been included as a reference. ZHYBRID simulations with two different outlet pressures have been presented in order to investigate the effect of shock intensity on the corner separation bubbles and the flow downstream of the shock in the SBLI region.

The simulations clearly indicate that the onset of the corner separation bubble relative to the shock location is one of the key issues for accurately predicting the SBLI flow. Moreover, the incoming boundary layer, the shock intensity and the shock foot shape are closely interconnected and an accurate prediction of these SBLI flow properties is challenging using hybrid RANS-LES simulations.

The ZHYBRID-a simulation has produced a $\lambda$-shape shock foot and a pressure rise across the shock, that are in reasonable agreement with experimental data. The simulation has slightly exaggerated the corner separation bubbles, which subsequently has led to a more pronounced cross flow.

Large differences have been observed on how the models have simulated the corner flows. The SA-IDDES model adapted reasonably to the recirculating flow and resolved a large part of the corner flow in LES mode. The SA-DDES model, on the other hand, produced high levels of turbulent viscosity in the corner separation bubbles and has much less turbulent structures resolved. As compared to the ZHYBRID-a and the experimental data, the SA-IDDES predicted a weaker $\lambda$-foot, which caused a slightly less distinct pressure rise in the wall pressure distribution across the shock. In the ZHYBRID simulations, the RANS and LES zones were prescribed and the model has resolved a part of the corner separation bubbles in LES mode, leading to an improved SBLI flow prediction. 
As a continuation of this paper, a grid resolution study will be carried out in order to analyze the effect on the SBLI region. Moreover, an extended duct will be used in order to locate the outlet boundary farther downstream from the SBLI region.

Acknowledgements This work was founded by the Swedish Governmental Agency for Innovation Systems (VINNOVA) in the Swedish National Flight Research Program (NFFP, Contract No. 2009-01346 and 2013-01209) and Saab Aeronautics with support from the EU project ATAAC, Contract No. 233710. Computational resources were supported by the Swedish National Infrastructure for Computing (SNIC).

\section{References}

1. Arvidson, S.: Assessment and Some Improvements of Hybrid RANS-LES Methods. Licenciate thesis ISSN 1652-8565, 2013:01, Applied Mechanics, Chalmers University of Technology (2013)

2. Arvidson, S, Davidson, L., Peng, S.H.: Hybrid RANS-LES Modeling Using a Low-ReynoldsNumber $k-\omega$ Based Model. AIAA paper 2014-0225, National Harbour, Maryland (2014)

3. Arvidson S., Peng, S.H., Davidson, L.: Feasibility of hybrid RANS-LES of shock/boundarylayer interaction in a duct. In Fu S. et al., editor, Progress in Hybrid RANS-LES Modelling 117 of NNFM, pages 245-256. Springer (2012)

4. Bruce, P. J. K., Babinsky, H.: Unsteady shock wave dynamics. Journal of Fluid Mechanics 603, 463-473 (2008)

5. Bruce, P. J. K., Babinsky, H., Tartinville, B., Hirsch, C.: Corner Effect and Asymmetri in Transonic Channel Flows. AIAA Journal 49, 2382-2392 (2011)

6. Eliasson, P.: EDGE, a Navier-Stokes Slover for Unstructured Grids. Scientific report FOI-R0298-SE, Computational Aerodynamics Department, Aeronautics Division, FOI (2001)

7. Menter, F.R.: Two-equation eddy-viscosity turbulence models for engineering applications. AIAA Journal 32, 1598-1605 (1994)

8. Gritskevich, M.S., Garbaruk, A.V., Schütze J., Menter F.: Development of DDES and IDDES Formulations for the $k-\omega$ Shear Stress Transport Model. Flow, Turbulence and Combustion 88, 431-449 (2012)

9. Peng, S.H., Davidson, L., Holmberg, S: A Modified Low-Reynolds-Number $k-\omega$ Model for Recirculating Flows. Journal of Fluids Engineering 119, 867-875 (1997)

10. Spalart P.R., Allmaras S.R.: A one-equation turbulence model for aerodynamic flows. La Recherche Aérospatiale 1, 5-21 (1994)

11. Shur, K.L, Spalart, P.R., Strelets, M.Kh., Travin, A.K.: A hybrid RANS-LES approach with delayed-DES and wall-modelled LES capabilities. International Journal of Heat and Fluid Flow 29, 1638-1649 (2008)

12. Spalart, P.R., Deck, S., Shur, M.L., Squires, K.D., Strelets, M. Kh., Travin, A: A new version of detached-eddy simulation, resistant to ambiguous grid densities. Theory of Computational Fluid Dynamics 20, 181-195 (2006) 